\title{
Das Bremerhavener Grundwasser im Klimawandel
}

\author{
Eine FREEWAT-Fallstudie
}

\author{
Björn Panteleit $^{1}$ (D) $\cdot$ Sven Jensen $^{1} \cdot$ Katherina Seiter $^{1} \cdot$ Yvonne Siebert $^{\text {Y }}$
}

Eingegangen: 6. September 2017 / Überarbeitet: 26. September 2017 / Online publiziert: 24. Januar 2018

(c) Der/die Autor(en) 2018. Dieser Artikel ist eine Open-Access-Publikation.

Zusammenfassung Ausgehend von einer umfangreichen Bohrdatenbank wurde für das Land Bremen ein 3D-Strukturmodell erstellt. Durch Interpretation und Interpolation der Bohrbeschriebe wurden den Modellzellen Parameter wie $\mathrm{k}_{\mathrm{f}}$-Werte zugewiesen. Diese Datenbasis des Strukturmodells wurde über die FREEWAT-Plattform, ein Opensource-Plugin der freien QGIS-Software, mit Anbindung des MODFLOW-Codes, in ein Strömungsmodell transferiert. Ziel war die Erstellung eines langfristig nutzbaren Werkzeugs zum Grundwassermanagement. Als FREEWATFallstudie wurden zunächst mögliche Auswirkungen prognostizierter Klimaveränderungen auf das Grundwasser im Bereich Bremerhaven berechnet. Neben dem Kalibrierungsjahr 2010, wurden für die Zeitschritte 2040, 2070, 2100 ein Meeresspiegelanstieg und eine abnehmende Grundwasserneubildung simuliert. Neben einer Meerwasserintrusion im Küstenbereich zeigen sich rückläufige Grundwasserdruckspiegel. Möglichkeiten eines zukünftigen Grundwassermanagements ergeben sich aus der Steuerung eines Seewasserspiegels und der Hafenbecken. Mithilfe eines auf den Modellergebnissen basierenden gezielten Grundwassermonitorings kann das vorgestellte Strömungsmodell im Rahmen fortgesetzter Modellpflege zukünftig ein wichtiges Prognosewerkzeug zum Grundwassermanagement bilden und Auswirkungen von veränderten Klimabedingungen und Maßnahmen darstellen.

Björn Panteleit

info@gdfb.de

1 Geologischer Dienst für Bremen, Leobener

Str. 8, 28359 Bremen, Deutschland

\section{The groundwater in Bremerhaven under climate} change

A FREEWAT case 3 study

Abstract A 3D structural model was created for the state of Bremen based on an extensive borehole database. Parameters were assigned to the model by interpretation and interpolation of the borehole descriptions. This structural model was transferred into a flow model via the FREEWAT platform, an open-source plug-in of the free QGIS software, with connection to the MODFLOW code. This groundwater management tool is intended for long-term use. As a case study for the FREEWAT Project, possible effects of climate change on groundwater levels in the Bremerhaven area have been simulated. In addition to the calibration year 2010, scenarios with a sea-level rise and decreasing groundwater recharge were simulated for the years 2040, 2070 and 2100. In addition to seawater intrusion in the coastal area, declining groundwater levels are also a concern. Possibilities for future groundwater management already include active control of the water level of a lake and the harbor basin. With the help of a focused groundwater monitoring program based on the model results, the planned flow model can become an important forecasting tool for groundwater management within the framework of the planned continuous model management and for representing the effects of changing climatic conditions and mitigation measures.

Keywords Climate change - Saltwater intrusion · MODFLOW · FREEWAT · Groundwater recharge 


\section{Einführung}

Während ca. $80 \%$ der Weltbevölkerung in den Küstenregionen dieser Welt leben (Chang et al. 2011) werden dort die örtlichen Küstenaquifere für die Versorgung des kommunalen, industriellen und landwirtschaftlichen Bedarfs genutzt (Konikow und Kendy 2005). Exzessive Aquifernutzung resultiert vielerorts in einer Aquiferverarmung mit abnehmenden Grundwasserspiegeln (Chang et al. 2011), was wiederum Salzwasserintrusionen und Upconing nach sich zieht und weitere Grundwasserverarmung bewirkt (Konikow und Kendy 2005). Viele der großen Aquifersysteme der Welt werden nicht-nachhaltigen Belastungen ausgesetzt und die Grundwasservorräte werden drastisch reduziert (Richey et al. 2015a). So sind auch die Grundwasserresourcen in Küstenaquiferen von weiterer Übernutzung und Kontamination (Barlow und Reichard 2010) bedroht. Obwohl eine nachhaltige Grundwassernutzung in Küstenstädten wie Bremerhaven eine entscheidende Rolle für das Aufrechterhaltung des ökologischen und wirtschaftlichen Standortes spielt (Barlow und Reichard 2010), gestaltet es sich aufgrund des Mangels an Daten und Kenntnissen um die Grundwasserprozesse noch immer schwierig, die mögliche, nachhaltig zu entnehmende Grundwassermenge verlässlich zu quantifizieren, wenn man von einer grundsätzlichen Resilienz des Grundwassersystems ausgeht (Richey et al. 2015b).

Als zusätzliche Belastung der Aquifersysteme werden in Zukunft Klimaveränderungen erwartet, die Auswirkungen auf alle Bereiche der Umwelt haben werden: Erwärmung der Atmosphäre und der Ozeane, abnehmende Schnee- und Eismassen, Anstiege des Meeresspiegels und Anstieg der Treibhausgaskonzentrationen (Stocker et al. 2013). Diese Veränderungen ziehen wiederum Auswirkungen auf Küstenaquifersysteme nach sich. Umso wichtiger ist es, das Verhalten von Aquifersystemen an Küsten unter verschiedenen Randbedingungen (einschließlich Klimaveränderungen) zu untersuchen, die ablaufenden Prozesse zu verstehen und die vorhandenen Grundwasserreserven zu evaluieren (Yang et al. 2015), um auch für die Zukunft eine nachhaltige Grundwasserversorgung zu gewährleisten. Während im Projekt KLIMU die Folgen einer Klimaänderung für den Grund- und Bodenwasserhaushalt der im Wesentlichen landwirtschaftlich genutzten Marsch westlich der Unterweser untersucht wurden (Hoffmann et al. 2006), liegen für den vorwiegend urban geprägten Bereich der Stadt Bremerhaven keine entsprechenden Untersuchungen vor.

Das hier vorgestellte Strömungsmodell soll langfristig als Werkzeug für ein nachhaltiges Grundwassermanagement im Bereich Bremerhaven dienen. Es basiert auf der umfangreichen Bohrdatenbank des Geologischen Dienstes für Bremen mit deren Informationen ein 3D-Strukturmodell mit räumlicher Verteilung von geologischen Parametern wie z. B. $\mathrm{k}_{\mathrm{f}}$-Werten erstellt wurde (Panteleit et al. 2013). Nach Transfer der Modellstruktur in ein MODLFOW-Strömungsmodell hat diese Arbeit das Ziel, Auswirkungen möglicher Klimaveränderungen auf die Grundwasserhydraulik und Grundwasserspiegelhöhen Bremerhavens zu simulieren. Die Simulation diverser Szenarien ist für die Zukunft ebenso geplant wie eine kontinuierliche Aktualisierung des Modells auf Basis neuer Bohrergebnisse und Wasserstandsmessungen.

\section{Freewat}

Die hier vorgestellten Untersuchungen stellen eines von 14 Fallbeispielen zu Anwendungsmöglichkeiten der im Rahmen des FREEWAT-Projektes (De Filippis et al. 2017) neu entwickelten Softwareplattform dar. FREEWAT ist ein HORIZON-2020-Projekt, finanziert durch die EU-Kommission im call „Water Innovation: Boosting is value for Europe“. Das Ziel des Projektes ist das Management von Wasserresourcen zu fördern. Durch den Einsatz erprobter moderner Simulationswerkzeuge soll die Anwendung der Wasserrahmenrichtlinie (Europäische Union 2000) und anderer EU-Wasserrichtlinien vereinfacht werden. Hierzu wurde im FREEWAT-Projekt eine Open-source- und Public-domain-Programmplattform entwickelt. Dieses GISintegrierte Management- und Planungsmodul bietet unter anderem eine Modellierungsumgebung zur Simulation der Quantität und Qualität von Oberflächenwasser als auch von Grundwasser.

Diese Ziele wurden in einem Plugin zur Open-sourceGIS-Software QGIS (QGIS 2015) umgesetzt. Das Plugin beinhaltet ein Modul AkvaGIS zur grafischen Präsentation und Auswertung des Wasserchemismus in vielfältigen Darstellungen z.B. Piper-, Stiff- oder SAR-Diagrammen. Als Simulationsmodule sind die bekannten Programme MODFLOW (in den Versionen 2005 (Harbaugh 2005) und OWHM (Hanson et al. 2014)), MT3DMS (Zheng und Wang 1999) und SEAWAT (Langevin et al. 2007) eingebunden. Zur Kalibrierung und Analyse stehen die Programme MODPATH (Pollock 1994), UCODE (Poeter et al. 2005) und ZONEBUDGET (Harbaugh 1990) zur Verfügung.

\section{Standort}

Der Fokus der Untersuchungen liegt im Bereich der Stadt Bremerhaven im Nordwesten Deutschlands am östlichen Rand des Weserestuars (Abb. 1). Bremerhaven hat ca. 108.000 Einwohner auf einer Fläche von ca. $94 \mathrm{~km}^{2}$. Zusammen mit den angrenzenden Gebieten ist das Modellgebiet $447,5 \mathrm{~km}^{2}$ groß. Es erstreckt sich $17,9 \mathrm{~km}$ in $\mathrm{E}-\mathrm{W}$-Ausdehnung und $25 \mathrm{~km}$ in N-S-Ausdehnung. Der aktive Modellrand orientiert sich an abgeleiteten Grundwasserscheiden der HÜK 200 (Dörhöfer et al. 2001). Das 
Abb. 1 Lage des Modellgebietes, Randbedingungen und Modelloberkante aus dem Digitalen Geländemodell, $A$ : Apeler See, $B$ : Ahnthammsmoor, $C$ : Wasserwerk Wulsdorf

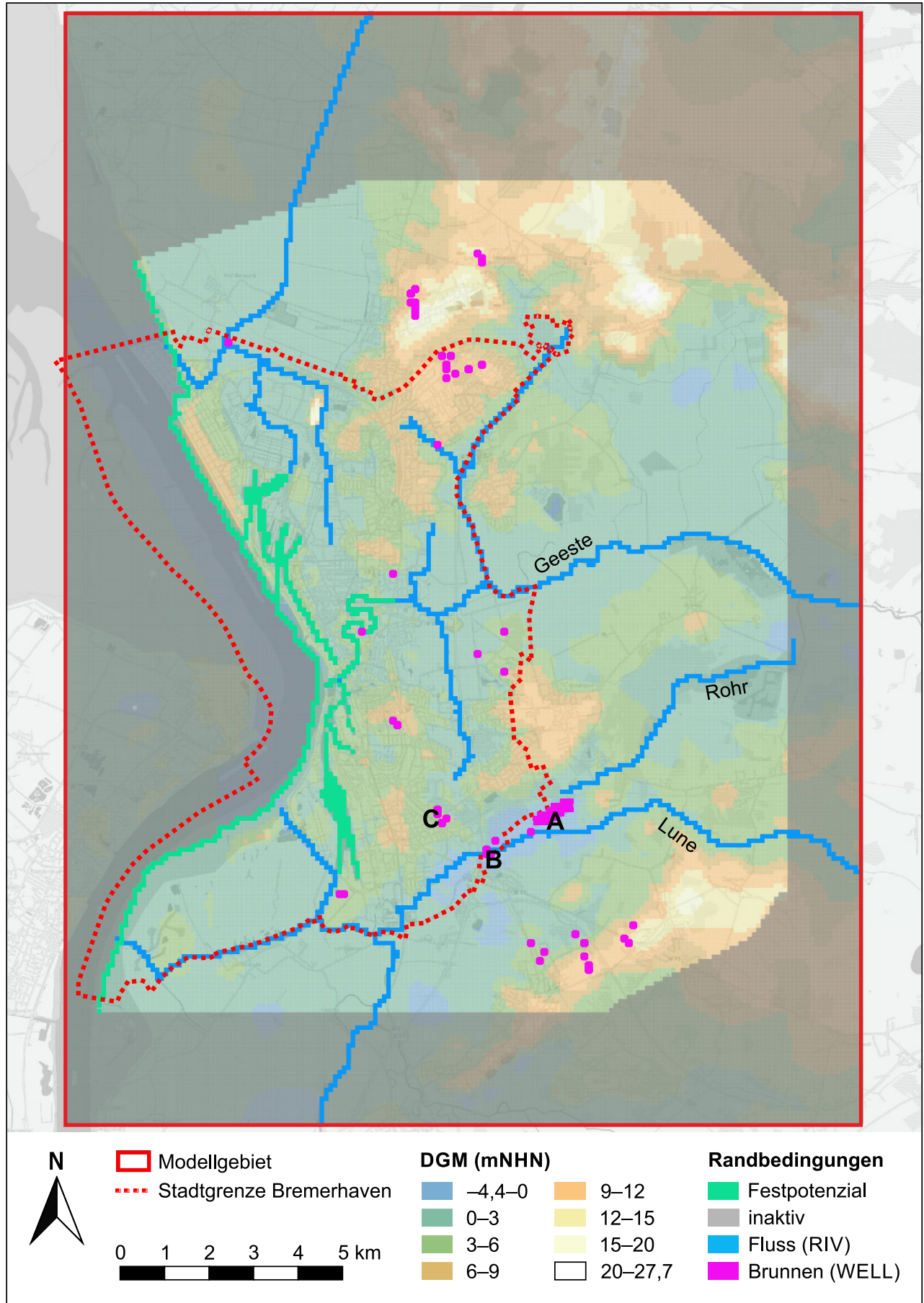

Untersuchungsgebiet hat seinen Ursprung in $3.466 .100 \mathrm{~m}$, 5.949.000 m (Rechtswert (Min), Hochwert (Max) im GaußKrüger Zone 3-Koordinatensystem).

Außerhalb des urbanen Bereichs finden sich landwirtschaftliche Flächen und Schutzgebiete sowie drei Wasserwerke, die die Stadt mit Trinkwasser versorgen. Bremerhaven und das umliegende Untersuchungsgebiet sind Teil des nord- und mitteldeutschen Lockergesteinsgebietes (Adhoc-AG Hydrogeologie 2016). Es teilt sich auf in die We- sermarsch mit Geländehöhen zwischen -2 und $6 \mathrm{mNN}$ und die höher liegenden Bereiche der Bederkesa-Geest (Geländehöhe zwischen 6 und 27,7 mNN) (Abb. 1).

Die Landschaft und der geologische Aufbau wurden vornehmlich durch das letzte Eiszeitalter, das Pleistozän, geprägt. Die Wechsellagerung sandiger und toniger Sedimente der Elster- und Saale-Kaltzeiten, die teilweise nur Linsenhaft erhalten sind, reichen bis in Tiefen von $240 \mathrm{~m}$. In den Geestbereichen sind Moränenablagerungen der Saale- 
Kaltzeit erhalten, während diese in den Marschbereichen durch Schmelzwässer der Weichsel-Kaltzeit erodiert wurden. In den entstandenen Niederungen kam es in der Folge zu Überschwemmungen, sodass die sandigen Grundwasserleiter durch organikreiche Tone (Auelehm) und Torfe bedeckt wurden.

Das betrachtete oberste Grundwasserstockwerk wird durch sechs hydrostratigraphische Einheiten aufgebaut. Den obersten Grundwasserleiter bilden Sande der SaaleKaltzeit (Jensen et al. 2003). Im Westen besteht ein hydraulischer Kontakt zur Weser, daran angrenzend überlagern bis zu $20 \mathrm{~m}$ mächtige Auelehme den Grundwasserleiter. Diese keilen nach Osten hin aus, und der Grundwasserleiter ist an kleinere Vorfluter, Seen und Drainagegräben angebunden. Abhängig von den überlagernden Auelehmen des Holozäns steht das Grundwasser in den Sanden der Saale-Kaltzeit frei oder gespannt an. Es ist teilweise versalzen aufgrund von Salzwasserintrusion im Küstenbereich oder historischen Überschwemmungen (Elbracht et al. 2016).

Der tiefere Grundwasserleiter liegt in den Sanden der Elster-Kaltzeit und wird lokal durch Feinsande aus dem Miozän und Pliozän ergänzt. Begrenzt wird das obere Grundwasserstockwerk durch mächtige bindige tertiäre Tone der Reinbeck- bzw. Langenfelde-Stufe. In ganz Norddeutschland haben subglaziale Schmelzwasser-Rinnen in die tertiäre Basis erodiert, die weitgehend NordSüd ausgerichtet sind. Drei dieser Rinnen (Bremerhavener, Ahnthammsmoorer und Bexhöveder Rinne) finden sich auch im Untersuchungsgebiet. Die Basis der Rinnen ist oft mit hydraulisch gut leitfähigen Sanden der noch hochenergetischen Elster-Kaltzeit gefüllt, während der obere Bereich durch die feinen bindigen Stillwasserablagerungen der jüngeren Elster-Kaltzeit, den sogenannten Lauenburger Schichten verfüllt wurde, sodass nur ein geringer hydraulischer Kontakt zu den angrenzenden Grundwasserleitern besteht (Elbracht et al. 2016). Außerhalb der Rinnen sind der Saale-kaltzeitliche und Elster-kaltzeitliche Grundwasserleiter lokal durch Lauenburger Ton oder Elster-kaltzeitlichen Geschiebelehm getrennt, die beide jedoch nur eine linsenartige Verbreitung aufweisen. In weiten Teilen des Untersuchungsgebiets treten die Lauenburger Schichten in ihrer feinsandigen Fazies auf (Ritterhuder Sande) und erlauben eine gute hydraulische Kommunikation der beiden Grundwasserleiter.

Die Fließrichtung des Grundwassers ist im Wesentlichen von den Neubildungsgebieten in den höher gelegenen Geest-Bereichen im Nordosten und Südosten des Untersuchungsgebietes in die Niederungen und letztendlich in die Weser gerichtet, die den westlichen Rand des Betrachtungsraumes bildet. Die Flurabstände liegen zwischen 2 und $25 \mathrm{~m}$.

\section{Methoden}

\section{Strukturbasierte Strömungsmodellierung}

Die Strömungsmodellierung basiert beim GDfB auf einem hydrostratigraphischen Strukturmodell, das mit der Software GOCAD (Mallet 1992) erstellt wurde (Panteleit et al. 2013). Zur Erstellung des Strukturmodells des Bereichs Bremerhaven wurde mithilfe der Bohrdatenbank des GDfB ein Gitter von 11 Nord-Süd und 26 West-Ost verlaufenden Profilschnitten erstellt. Die Basishorizonte der hydrostratigraphischen Einheiten in diesen Profilschnitten wurden mithilfe zusätzlicher Punktdaten der Basislage der Einheiten aus ausgewählten Bohrungen in die Fläche interpoliert. Aus den Basishorizonten der hydrostratigraphischen Einheiten in $100 \times 100 \mathrm{~m}$ Raster-Auflösung wurden Volumenkörper (SGRIDs) erstellt. Die so erhaltenen Körper der hydrostratigraphischen Einheiten sind jedoch in sich nicht homogen ausgebildet, sondern können petrographische Variationen aufweisen, die oftmals auch zu linsenförmigen Strukturen führen.

Um derartige lokale Sedimentwechsel innerhalb der Einheiten wie z.B. Geschiebelehmlinsen in den Elster-kaltzeitlichen Ablagerungen im Strukturmodell abbilden zu können, wurden die Einheiten in mehrere Schichten unterteilt, deren Mächtigkeit innerhalb der Einheit konstant ist. Die Anzahl der Schichten pro Einheit wurde hierbei so gewählt, dass die Mächtigkeit der Linsenstrukturen in den einzelnen Einheiten möglichst gut abgebildet werden kann und die Gesamtzahl der Schichten nicht unnötig groß wird. Um die Anforderung von MODFLOW von durchgehenden Modellschichten in der späteren Strömungsmodellierung zu berücksichtigen, wurden alle Schichten über das gesamte Modell durchgehend mit einer Mindestmächtigkeit von $0,1 \mathrm{~m}$ erstellt. Das resultierende Schichtmodell ist im östlichen Anschnitt in der Abb. 2 und der Abb. 3 zu entnehmen. Hierzu wurde den einzelnen Schichten der verfügbaren 16.254 archivierten Bohrungen auf Basis des Petrographiebeschriebs mithilfe der Software GCI-Petro- $\mathrm{k}_{\mathrm{f}}$ (Fuchs 2010) ein $\mathrm{k}_{\mathrm{f}}$-Wert zugewiesen. Den durch eine archivierte Bohrung aufgeschlossenen Zellen wurde sodann der gemittelte $\mathrm{k}_{\mathrm{f}}$-Wert der jeweiligen Schichten zugewiesen und diese über eine Interpolation auf das gesamte Modellraster übertragen. Auskeilende Schichten können in diesem Modellgitter somit durch eine entsprechende Parametrisierung durch Zuweisung der tatsächlich in der entsprechenden Tiefe angetroffenen Parameter berücksichtigt werden. Linsenstrukturen ergeben sich, wie im südlichen Modellanschnitt in Abb. 2 ersichtlich, ebenfalls durch eine Zuweisung der entsprechenden Parameter in einigen Zellen der Einheit. Die resultierenden Strukturen wie z. B. Bereiche mit gering durchlässigen Geschiebelehmlinsen innerhalb der Einheit der Elster-Kaltzeit haben eine hohe Übereinstimmung mit 
Abb. 2 Strukturmodell: im östlichen Anschnitt mit den Hydrostratigrafischen Einheitiefer liegenden als Basis für die Strömungsmodellierung; im südlichen Anschnitt mit den zugewiesenen $\mathrm{k}_{\mathrm{f}}$-Werten, die auch eine Wiedergabe von Linsenstrukturen im Bereich der Rinnen erkennen lassen ten und Modellgitter in den

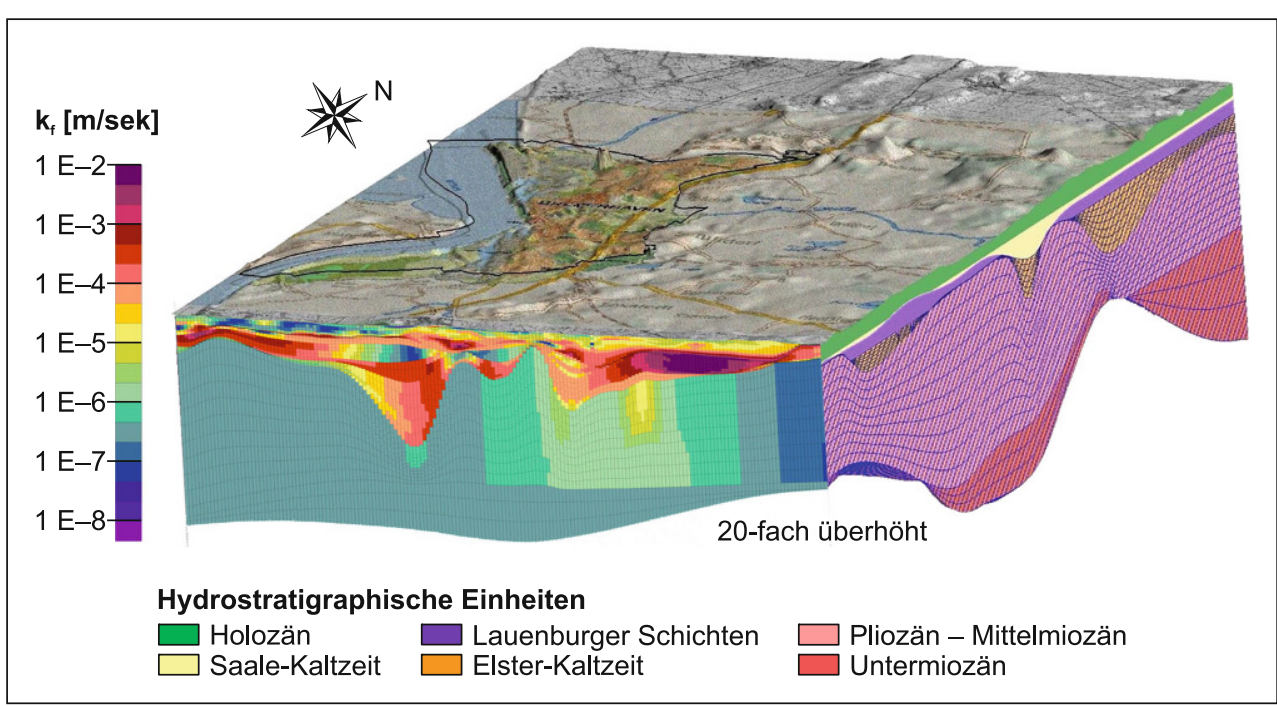

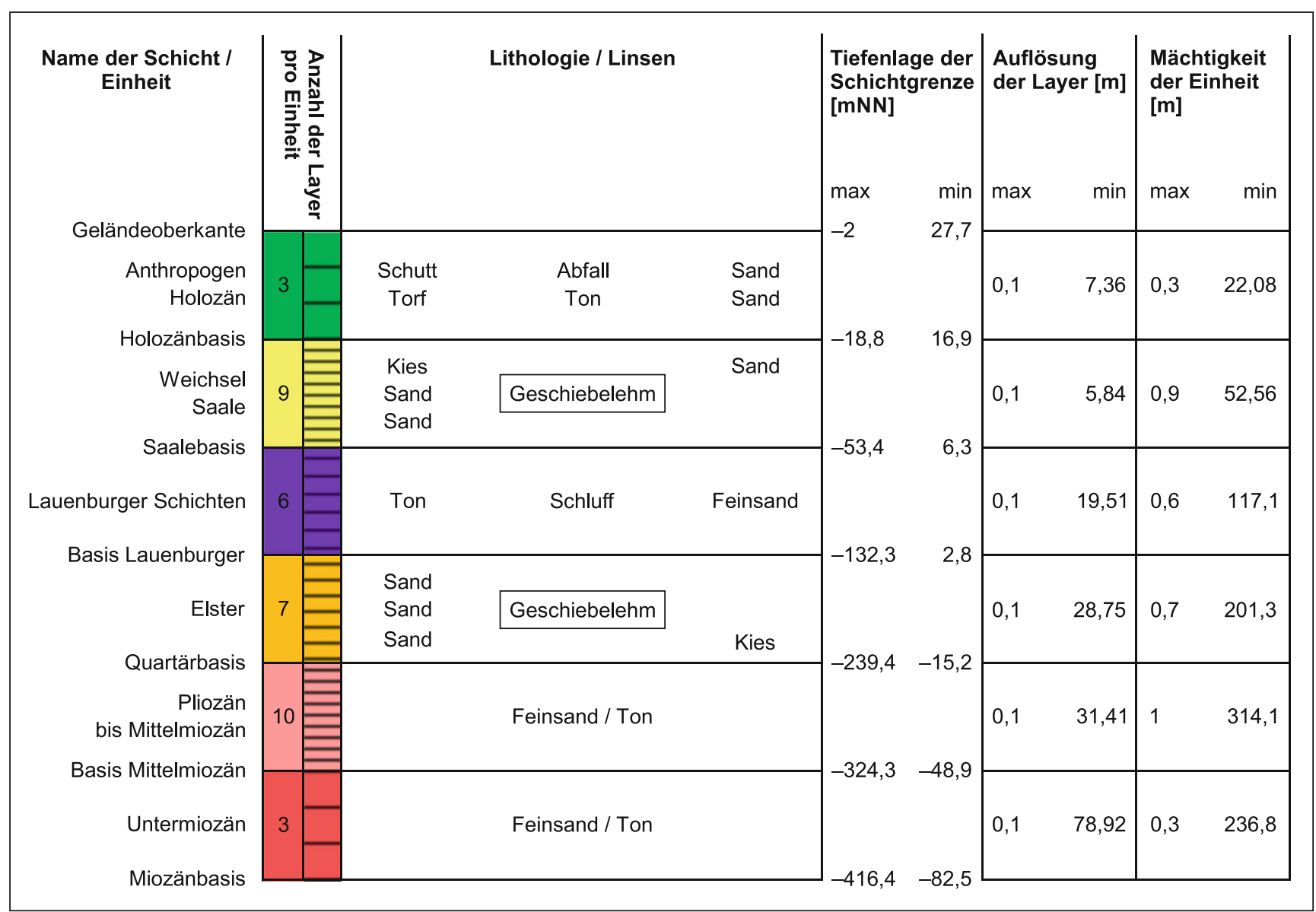

Abb. 3 Aufbau des Strukturmodells, Farben korrespondieren mit der Wiedergabe im Strukturmodell (Abb. 2) 
den geologischen Strukturen. Dies ist ein wesentlicher Unterschied zu Strömungsmodellen mit einer geringen Anzahl von Schichten, die nur eine über die Schichtmächtigkeit gemittelte Transmissivität wiedergeben können.

Die $\mathrm{k}_{\mathrm{f}}$-Werte stellen in dem so erhaltenen Gitter für die Strömungsmodellierung also keine freie Kalibrierungsgröße mehr dar, sondern geben in ihrer Struktur die geologischen Verhältnisse wieder.

Es wird ein stationäres Modell aufgebaut und auf Daten einer Stichtagsmessung aus dem Herbst 2012 kalibriert. Diese Daten spiegeln ein langjähriges Mittel wider und stellen darüber hinaus die breiteste zur Verfügung stehende Datengrundlage dar.

\section{Hydraulischer Modellaufbau}

Über eine ASCII-Ausgabe der Modellschichten wurde das auf oben genanntem Weg erstellte Modellgitter mithilfe eines Phytonscripts in QGIS importiert und um die hydraulischen Randbedingungen ergänzt. Hierzu stehen Wasserstandsdaten von Pegeln der Oberflächengewässer aus einer Stichtagsmessung aus dem Herbst 2012 zur Verfügung. Der Zielsetzung folgend ein regionales Übersichtsmodell zu erstellen, wurden hier nur größere Oberflächengewässer berücksichtigt. Kleinere Entwässerungsgräben, die im Gebiet vorhanden sind, wurden im Modell nicht eingebaut. Ergänzt wurden diese Informationen durch die Jahresentnahmemengen der im Modellgebiet vorhandenen Förderbrunnen für das Jahr 2012.

An den Rändern des aktiven Modellbereichs im Norden, Süden und Osten erfolgt kein Grundwasserzufluss (NoFlow Boundaries), da diese parallel zur Grundwasserfließrichtung bzw. an Grundwasserscheiden in den Geestgebieten im Nordosten und Südosten gewählt wurden. Der resultierende aktive Modellbereich hat eine Fläche von rund $233 \mathrm{~km}^{2}$ mit 886.008 Zellen (in 38 Layern). Er enthält

- 78 Modellzellen mit einer Grundwasserentnahme durch Förderbrunnen, die überwiegend in den Geestbereichen liegen und zur Rohwasserversorgung für die öffentliche Trinkwasserversorgung dienen. Einige industrielle Entnahmen befinden sich jedoch auch in den an die Weser angrenzenden Gewerbegebieten.

- 13 Wasserläufe, die über das Modflow-River-Package in das Modell eingebunden werden.
- 429 Grundwassermessstellen, die mit mindestens jährlichen Stichtagsdaten zur Verifizierung der modellierten Wasserstände zur Verfügung stehen.

- eine flächenhafte Grundwasserentnahme im Apeler See, dessen Wasserstand über ein Schöpfwerk geregelt wird.

\section{Klimaszenarien}

Die möglichen Auswirkungen von Klimaänderungen auf das Grundwasser im Bereich Bremerhaven wurden für vier Zeitstufen (die Jahre 2010, 2040, 2070 und 2100) eines Klimaszenarios simuliert. Hierfür wurden die klimaabhängigen Randbedingungen der Meeresspiegelhöhe und der Grundwasserneubildung für die verschiedenen Zeitstufen variiert (s. Tab. 1).

Für die Unterstützung von Entscheidungsträgern und Planern ist das Ergebnis von Worst-case-Simulationen der entscheidende Wert. Dementsprechend wurden in dieser Arbeit die Meeresspiegelanstiegsraten aus dem Worst-caseSzenarium RCP8.5 des IPCC mit den höchsten Treibhausgasemissionsraten und den höchsten Meeresspiegelanstiegsraten abgeleitet.

Der globale mittlere Meeresspiegelanstieg wird nach dem IPCC AR5 (IPCC 2013) nahezu sicher weiter fortschreiten. Mit einer mittleren Sicherheit im 95\% Konfidenzintervall wird der globale Meeresspiegelanstieg mit Prozess-basierten Modellen im Worst-case-Szenarium RCP8.5 des IPCC AR5 bis zum Ende des 21. Jahrhunderts bei 0,52 bis $0,98 \mathrm{~m}$ liegen. Auch Schirmer (2006) geht für den westlichen Unterweserbereich davon aus, dass die Worst-case-Variante für das Klimaszenario der Fallstudie „Klimaänderung und Unterweserregion“ (KLIMU) die relevanten Werte für mögliche Szenarien liefert. Für die vorliegenden Berechnungen wurde eine mittlere Meeresspiegelanstiegsrate dieses Worst-case-Szenarios gewählt, sodass sich die in Tab. 1 aufgeführten Meeresspiegelhöhen für die vier Zeitstufen ergeben.

Die Grundwasserneubildung wird sowohl durch eine aus prognostizierten Temperaturveränderungen resultierende erhöhte Evapotranspiration als auch durch eine veränderte Niederschlagsverteilung mit einhergehenden variierenden Oberflächenabflussraten beeinflusst. Die Daten der Grundwasserneubildungsraten für die gewählten vier Zeitstufen wurden, basierend auf Daten der WETTREG-2010 R4-Kli-

Tab. 1 Meeresspiegelanstieg und Grundwasserneubildung im Modellgebiet

\begin{tabular}{lllll} 
Jahr & 2010 & 2040 & 2070 & 2100 \\
\hline Mittlere GW-Neubildung & $150 \mathrm{~mm} / \mathrm{Jahr}$ & $147 \mathrm{~mm} / \mathrm{Jahr}$ & $105 \mathrm{~mm} / \mathrm{Jahr}$ & $62 \mathrm{~mm} / \mathrm{Jahr}$ \\
$\begin{array}{l}\text { Gesamte GW-Neubil- } \\
\text { dung im Modell/Jahr }\end{array}$ & $34.974 \mathrm{Miom}^{3}$ & $34.274 \mathrm{Miom}^{3}$ & $24.481 \mathrm{Miom}^{3}$ & $14.456 \mathrm{Miom}^{3}$ \\
Meeresspiegel & $0 \mathrm{mNHN}$ & $0,18 \mathrm{mNHN}$ & $0,45 \mathrm{mNHN}$ & $0,78 \mathrm{mNHN}$ \\
\hline
\end{tabular}




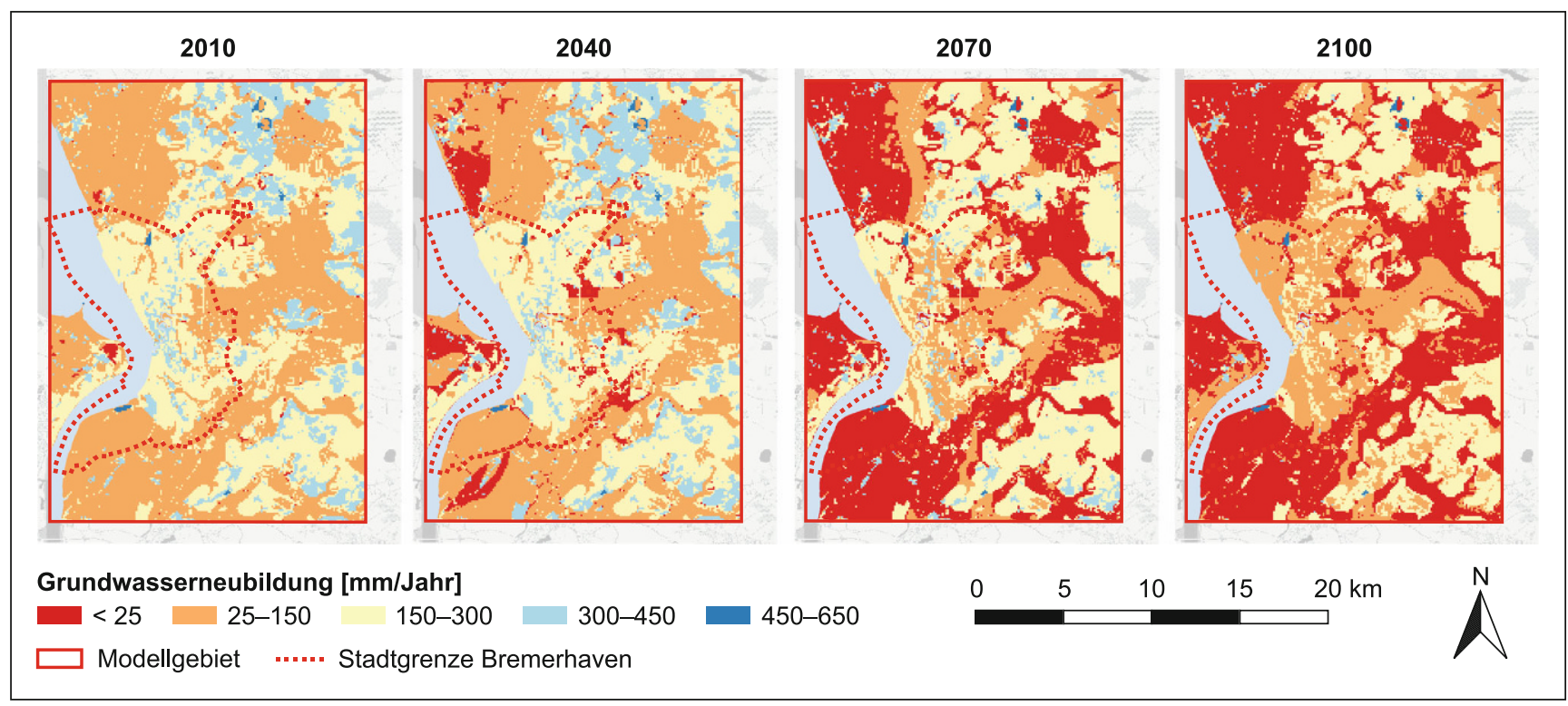

Abb. 4 Grundwasserneubildung für die ausgewählten Szenarien

mamodelle für die Modellierungen mit dem Wasserhaushaltsmodell mGROWA (Herrmann et al. 2013), variiert. Hierfür haben Herrmann et al. (2013) die Grundwasserneubildungsraten für Niedersachsen und Bremen in vier Simulationszeiträumen mit je 30 Jahren projiziert. Diese zeigen für den Raum Bremerhaven eine Abnahme der jährlichen Gebietsgrundwasserneubildung bis zum Jahr 2100 (Abb. 4).

\section{Kalibrierung und Verifizierung}

Für die Modellkalibrierung stehen Wasserstandsdaten von Grundwassermessstellen aus dem Herbst 2012 zur Verfügung. In einem ersten Schritt werden die aus den Schichtenverzeichnissen interpretierten und interpolierten $\mathrm{k}_{\mathrm{f}}$-Werte unverändert in das Modell implementiert und die resulitierenden Modellergebnisse mit den Messwerten verglichen.

Um die Verbindung zum Strukturmodell, auch für eine geplante zukünftige Modellpflege mit neuen Bohrdaten, zu erhalten, erfolgt die Modellkalibrierung im Wesentlichen über eine für das gesamte Modell einheitliche Variation der $\mathrm{k}_{\mathrm{f}}$-Werte. Die in die FREEWAT-Plattform ebenfalls integrierte Kalibrierung durch regionale Variationen von $\mathrm{k}_{\mathrm{f}}{ }^{-}$ Werten mit UCODE wird an dieser Stelle daher nicht genutzt.

Um mögliche systematische Über- oder Unterschätzungen durch die verwendete Software zur $\mathrm{k}_{\mathrm{f}}$-Werte-Zuweisung $\mathrm{zu}$ minimieren, werden die $\mathrm{k}_{\mathrm{f}}$-Werte zunächst insgesamt vermindert bzw. vergrößert. Hier ergeben sich die plausibelsten Ergebnisse, wenn die im Rahmen der Strukturmodellierung ermittelten $\mathrm{k}_{\mathrm{f}}$-Werte insgesamt um den Faktor 1,95 erhöht werden.
Im nächsten Schritt erfolgte eine weitere Anpassung durch die Veränderung des Verhältnisses der horizontalen Durchlässigkeit zur vertikalen Durchlässigkeit. Hierbei bleiben die horizontalen $\mathrm{k}_{\mathrm{f}}$-Werte jetzt unverändert, die vertikalen $\mathrm{k}_{\mathrm{f}}$-Werte werden insgesamt weiter variiert. Es wird dabei davon ausgegangen, dass diese nicht geringer als ein Zehntel der horizontalen $\mathrm{k}_{\mathrm{f}}$-Werte sind (Langguth und Voigt 2004) und, nach Auswertungen von Laborversuchen, den horizontalen $\mathrm{k}_{\mathrm{f}}$-Werten im Extremfall entsprechen können (Entenmann 1998). In diesen vorgegebenen Grenzen werden die vertikalen $\mathrm{k}_{\mathrm{f}}$-Werte durch manuelle Iteration solange variiert, bis die Angleichung von modellierten Grundwasserständen an gemessene Grundwasserstände nicht weiter zu verbessern ist. Als Ergebnis dieser Kalibrierung ergibt sich hier für das Modellgebiet ein Verhältnis zwischen horizontaler und vertikaler Durchlässigkeit von 0,89 .

Ein zusätzlicher Schritt der Kalibrierung ist die Anbindung der Oberflächengewässer an den Grundwasserleiter. Hier wurde nach dem Try-and error-Verfahren die Anbindung ermittelt, welche die geringsten Abweichungen zwischen gemessenen und berechneten Grundwasserständen in beeinflussten Grundwassermessstellen zur Folge hat.

Eine Verifizierung erfolgt zum einen über eine Darstellung der für die verwendeten Messstellen modellierten Grundwasserstände im Verhältnis zu den dort gemessenen Grundwasserständen und eine Berechnung des Verhältnisses des Quadratischen Mittels dieser Abweichungen zur Gesamtpotenzialdifferenz. Darüber hinaus wird ein auf Grundlage der Messwerte konstruierter Grundwassergleichenplan zum modellierten Grundwassergleichenplan ins Verhältnis gesetzt (Abb. 5). Der Einfluss durch erhöh- 


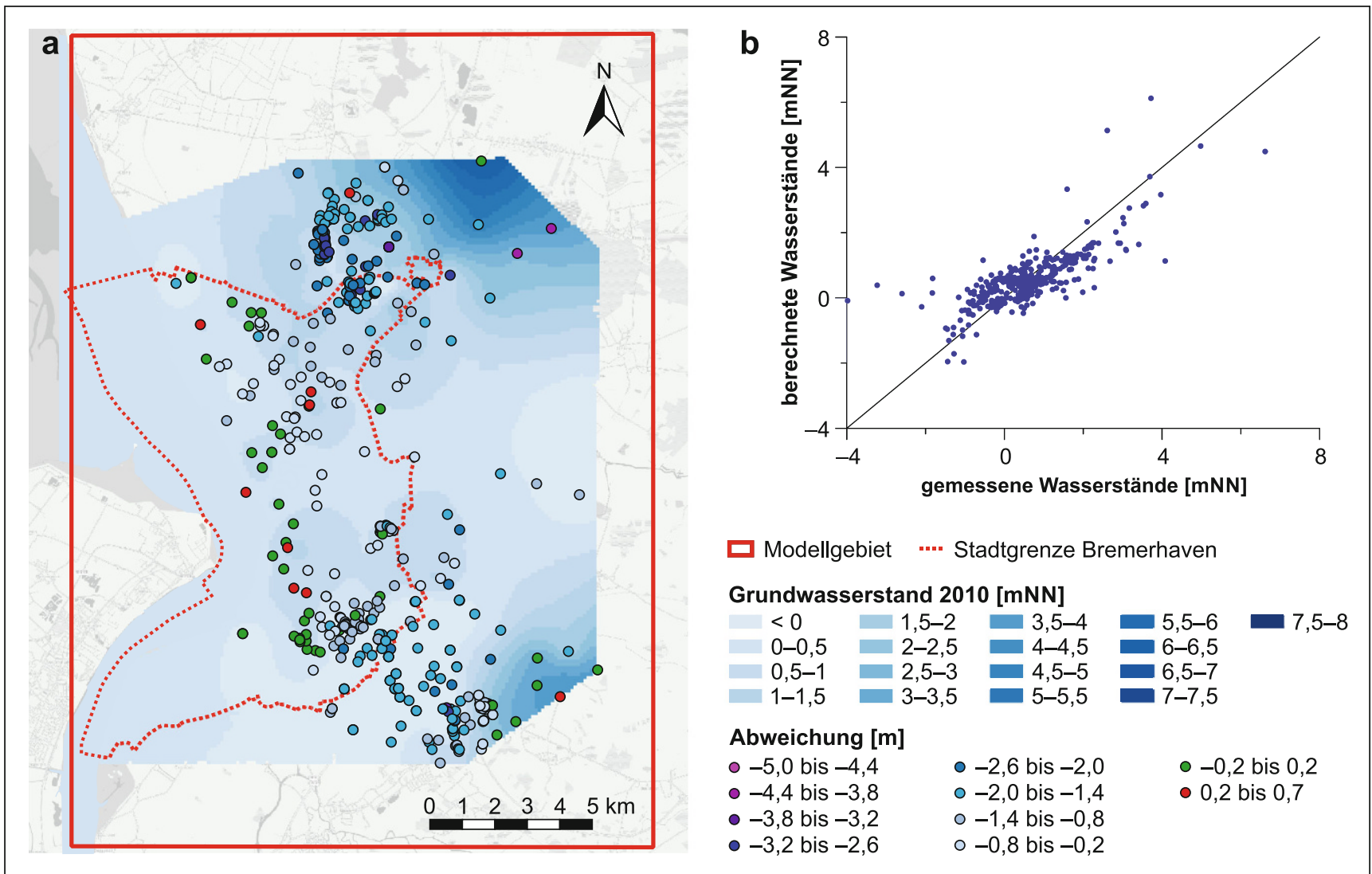

Abb. 5 Korrelation zwischen Messwerten und modellierten Werten; a auf Basis einer Stichtagsmessung erstellter Grundwassergleichenplan mit Abweichung zum Modellergebnis in den Messstellen; b Crossplot der gemessenen und berechneten Grundwasserstände

te Dichte in durch Salzwasser beeinflusste küstennahen Grundwassermessstellen ist nach Post et al. (2007) aufgrund der geringen Filtertiefen mit weniger als $5 \mathrm{~cm}$ zu erwarten.

Es ergibt sich eine Überschätzung der berechneten Grundwasserstände von maximal $5 \mathrm{~m}$ und eine Unterschätzung von höchstens $0,7 \mathrm{~m}$ gegenüber den Messwerten. Daraus ergibt sich über das Modellgebiet eine Varianz von 0,5. Die Wurzel der mittleren quadratischen Abweichung zwischen gemessenen und berechneten Wasserständen beträgt 0,71 . Dies entspricht $6,7 \%$ der gemessenen Gesamtpotenzialdifferenz $(-3,98$ bis $6,62 \mathrm{mNN})$.

Die für das Jahr 2010 berechneten Grundwasserstände sind in der Abb. 6 dargestellt. Der minimale Grundwasserstand liegt bei $-3,17 \mathrm{mNN}$ und tritt in einer einzelnen Modellzelle eines Entnahmebrunnens im Bereich der Trinkwasserfassungsanlage Ahnthammsmoor auf. Aufgrund der relativ großen Modellzellen sind weitere Absenkbereiche durch Grundwasserentnahmen in den Modellergebnissen kaum erkennbar. So liegen in den übrigen Modellbereichen die Wasserstände zwischen ca. $-0,77$ und $11,28 \mathrm{mNN}$. Die Hochlagen der Grundwasseroberfläche befinden sich erwartungsgemäß in den Geestbereichen, die eine erhöhte Grundwasserneubildung erfahren. Von dort besteht ein Gefälle in
Richtung der als Vorfluter wirkenden, von Ost nach West, fließenden Flüsse Geeste und Lune sowie der Weser am westlichen Rand des Modellbereiches.

Die Grundwasserstände für das kalibrierte Modell (Zeitstufe für das Jahr 2010) zeigen eine gute Übereinstimmung mit den gemessenen Werten aus dem Jahr 2012. Als quantitative Größe kann hierfür die Wurzel der mittleren quadratischen Abweichungen (QMW) zwischen gemessenen und berechneten Werten im Verhältnis zur Gesamtpotenzialdifferenz gesehen werden. Diese sollte nach (Anderson und Woessner 1992) möglichst klein sein. Ein Zielwert von 5\% wird hier allgemein angestrebt. Für dieses regionale Modell wurde eine homogene Zellgröße von $100 \times 100$ m gewählt, mit dem Absenktrichter im Bereich der vier Wasserwerke nicht detailliert abgebildet werden können. So kommt es naturgemäß in diesen Bereichen zu größeren Abweichungen zwischen den berechneten Grundwasserständen und den Messwerten in den im näheren Umfeld der Entnahmebrunnen gelegenen Grundwassermessstellen. Teilweise liegen hier mehrere Messstellen in einer Modellzelle. Vor diesem Hintergrund erscheint die Kalibrierung zufriedenstellend, auch wenn der für das vorliegende Modell erzielte Wert für QMW/Gesamtpotenzialdifferenz von 6,7\% über dem genannten Zielwert liegt. Dies zeigt auch ein Vergleich 


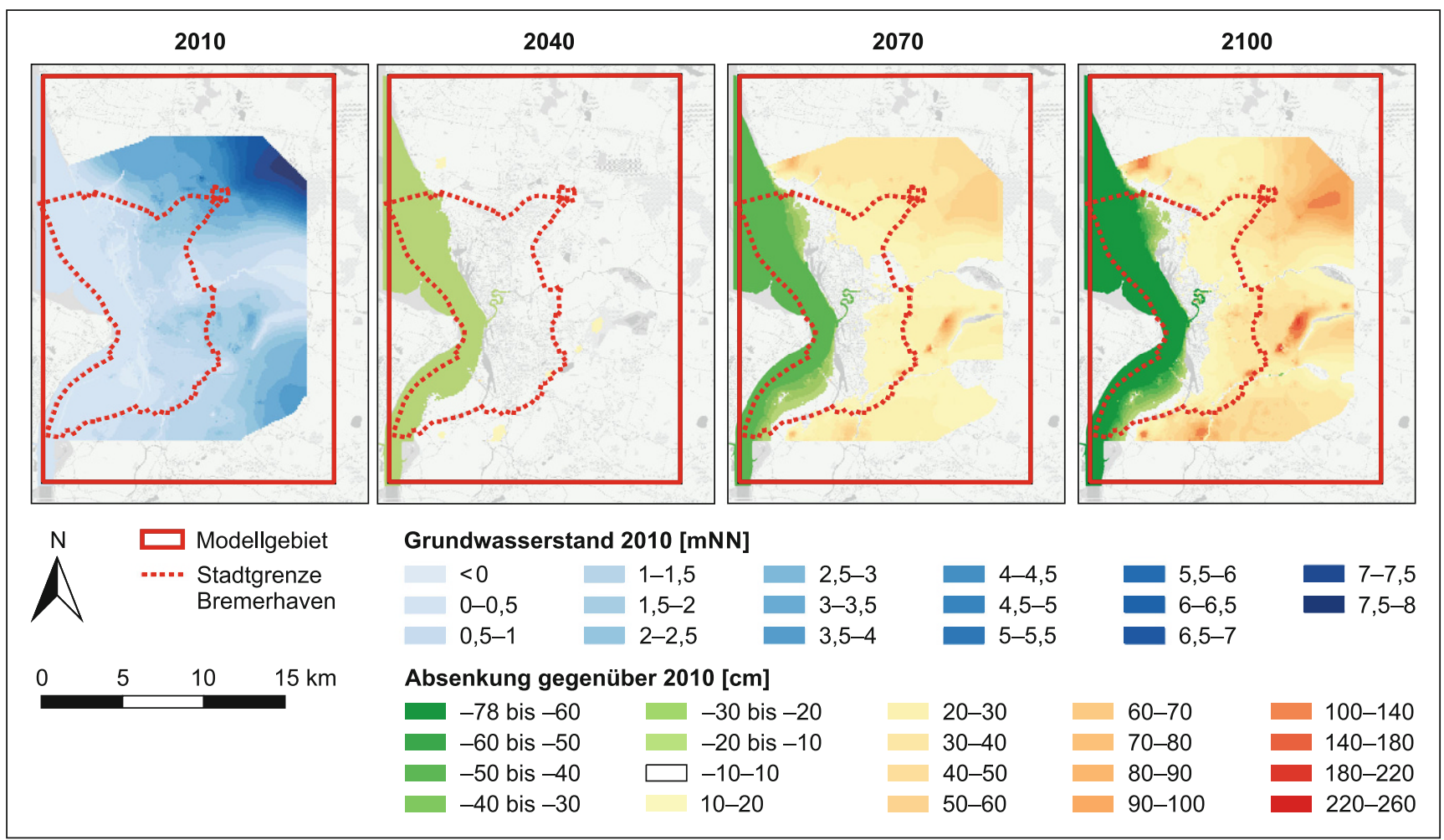

Abb. 6 Ergebnis des kalibrierten Modells und Differenzendarstellung für die untersuchten Szenarien

des berechneten Grundwassergleichenplans mit einem auf Basis der Messwerte erstellten Gleichenplan, der keine gröBeren Abweichungen in Gefälle und Fließrichtung erkennen lässt (Abb. 5 und 6). Beim Vergleich der Daten zeigt sich in den modellierten Grundwasserständen der von den Vorflutern durchflossenen Niederungen ein höherer Detaillierungsgrad. Dieser erscheint plausibel, da diese Bereiche eine sehr geringe Messstellendichte aufweisen.

Wird auf den Einbau und die Berücksichtigung von Messstellen in direkter Umgebung von Entnahmebrunnen verzichtet, verbessert sich die Varianz zwischen gemessenen und berechneten Wasserständen im Modell auf 0,39.

Eine weitergehende Modellverifizierung steht noch aus, hierfür soll eine erneute Stichtagsmessung durchgeführt werden, um die Veränderungen nach der Übertragung der Fördermengen des Wasserwerks Wulsdorf auf andere Wasserwerke im Modellgebiet im Jahr 2017 zu erfassen.

Es kann daher festgehalten werden, dass, zumindest im regionalen Maßstab, mit dem Ansatz der strukturbasierten Strömungsmodellierung, in der die Durchlässigkeitsbeiwerte mit einer nur geringfügigen und über das gesamte Modellgebiet einheitlichen Anpassung aus dem Beschrieb der Bohrprotokolle abgeleitet werden, auch ohne eine regionale Variation zu Kalibrierungszwecken, das Grundwasserfließregime erfolgreich abgebildet werden kann.

Abweichungen zwischen modellierten und gemessenen Grundwasserständen in Detailbereichen sollen im Zuge ei- ner weiteren Modellpflege minimiert werden. Eine Variation der Anbindung der Oberflächengewässer ebenso wie eine lokale Verfeinerung des Modellgitters in Bereichen einer hohen Informationsdichte bieten hierzu Möglichkeiten. Des Weiteren ist eine Überarbeitung der Grundwasserneubildungsdaten in Detailbereichen vonnöten. Hier können die hohen Werte im Bereich einer Deponie, deren Sickerwässer aufgefangen und abgeführt werden, ebenso zu Irritationen führen, wie die in der Prognose deutlich rückgängigen Neubildungsraten im Bereich Weser. Die Modellergebnisse beeinflussen diese Daten aufgrund ihrer Nähe zu den vorgegebenen Randbedingungen vermutlich jedoch nur unwesentlich.

\section{Klimaauswirkungen auf das Grundwasser}

Für die Modellberechnungen der prognostizierten Auswirkungen des Klimawandels sind die Veränderungen in den Grundwasserständen für die Jahre 2040, 2070 und 2100 in der Abb. 6 wiedergegeben.

Entsprechend der im ersten Zeitschritt geringen Reduzierung der Grundwasserneubildung werden hier in wenigen Bereichen um bis zu $0,35 \mathrm{~m}$ sinkende Grundwasserstände berechnet. Der im Festpotenzial der Weser berücksichtigte steigende Meeresspiegel von $0,18 \mathrm{~m}$ wirkt sich vor allem im Norden und Süden des Stadtgebietes aus. Im Norden 
reicht der Anstieg des Grundwasserstands von mindestens $0,1 \mathrm{~m}$ bis in ca. $800 \mathrm{~m}$ Entfernung, im Süden reicht dieser Anstieg bis in ca. $1200 \mathrm{~m}$ vom Weserufer. Hierbei handelt es sich um den Anstieg des Druckspiegels, da der Grundwasserleiter überwiegend durch Auelehm überlagert wird und dadurch gespannt ist.

Für das Modelljahr 2070 zeigen sich bereits stärkere Veränderungen in den Grundwasserständen. Die maximalen Absenkungen sind ca. 0,3 m höher und betreffen neben den Geestgebieten vor allem das Naturschutzgebiet Sellstedter See und Ochenstriftmoor/Wildes Moor nordöstlich des Apeler Sees in der östlichen Marsch zwischen den Vorflutern Geeste und Rohr. Der Anstieg des Grundwasserdruckspiegels im Nordwesten reicht nun ca. $1500 \mathrm{~m}$ ins Landesinnere, im Südwesten ist ein Anstieg von mehr als $0,1 \mathrm{~m}$ bis in $1800 \mathrm{~m}$ vom Weserufer entfernt festzustellen.

Der bereits für das Modelljahr 2070 festzustellende Trend setzt sich im Modelljahr 2100 verstärkt fort. Die maximalen Absenkungen betragen über $2 \mathrm{~m}$ und liegen im zuvor benannten Naturschutzgebiet. Von den Geestbereichen ist vor allem der nördliche Bereich mit Absenkbeträgen von über $1,0 \mathrm{~m}$ betroffen. Eine Grundwasserabsenkung von über $0,1 \mathrm{~m}$ ist jedoch über das gesamte Modellgebiet festzustellen und wird nur zur Küste hin durch den dort wirksamen Anstieg des Meeresspiegels abgepuffert. Dieser wirkt im Norden nunmehr $2100 \mathrm{~m}$ ins Landesinnere, während im Süden sich vor allem ein steilerer Gradient ausbildet und der Anstieg ca. $1900 \mathrm{~m}$ vom Weserufer durch die auf der Landesgrenze verlaufende Lune abgefangen wird.

Die Ergebnisse der untersuchten Szenarien zeigen aufgrund des Einflusses des steigenden Meeresspiegels bis 2100 steigende Grundwasserdruckspiegel. Im zentralen Bereich der Stadt wird dieser Effekt durch die konstant gehaltenen Wasserspiegel in den direkt angrenzenden abgeschleusten Hafenbecken aufgefangen. Ob dieser jedoch auch in der Realität gestiegenen Wasserstand der Weser auf dem derzeitigen Niveau gehalten wird ist fraglich. Offen ist auch die Güte der hydraulische Anbindung der Weser und des Hafenbeckens an den Grundwasserleiter, der zwar für den finalen Gleichgewichtszustand nicht erheblich ist, in der instationären Berechnung aber bestimmend ist für den Zeitpunkt, zu dem der Gleichgewichtszustand erreicht wird. Für die Stadt könnte der steigende Meeresspiegel weniger aufgrund des bereichsweise steigenden Druckspiegels problematisch sein, sondern vielmehr in Hinsicht auf die damit einhergehende Salzwasserintrusion. Während die Gewinnung von Trinkwasser in den Geestgebieten hiervon unbeeinflusst bleiben dürfte, sind auf der Abb. 1 auch einige industriell genutzte Förderbrunnen im Hafengebiet zu erkennen, die direkt betroffen wären. Eine Anpassung der Anlagen wassernutzender Betriebe auf veränderte Wasserqualitäten könnte erforderlich sein, sofern die Eigenförderung auch unter veränderten Randbedingungen aufrecht gehalten werden soll. Der Aspekt der Salzwasserintrusion soll zukünftig durch eine SEAWATModellierung näher untersucht werden. Aktuell ist dies für die Modellstruktur noch limitiert, da FREEWAT in der aktuellen Version 0.5 noch nicht die Festlegung der Zeitschritte bzw. der Frequenz der Ergebnisausgabe bietet. Aufgrund der Modellstruktur mit einer Schichtmächtigkeit von teilweise bis zu $0,1 \mathrm{~m}$ ergeben sich sehr kleine Zeitschritte in der Modellierung mit entsprechend nicht zu handhabenden Ergebnis-Files.

Während der Meeresspiegelanstieg sich naturgemäß in den küstennahen Bereichen im Westen des Modellgebietes abbildet, betrifft die insbesondere für die zweite Hälfte des Jahrhunderts prognostizierte Abnahme der Grundwasserneubildung vor allem die östlichen Bereiche (s. Abb. 6). Hier sinkt der Grundwasserdruckspiegel bis zu 2,6 $\mathrm{m}$ ab. Die Absenkungen sind dabei vor allem durch die lokale Grundwasserneubildung gesteuert, die in offenen Gewässern und Moor- bzw. Waldgebieten aufgrund der höher angesetzten Evapotranspiration deutlich zurückgeht. So zeigen sich die höchsten Auswirkungen im Bereich des Naturschutzgebietes Sellstedter See und Ochenstriftmoor/Wildes Moor, während die Trinkwassergewinnungsgebiete nur eine Absenkung des Grundwasserdruckspiegels im Bereich von 0,5 m erfahren. Die Fördermengen der Entnahmebrunnen wurden dabei in diesen Berechnungen konstant gehalten. Eine Verlagerung oder Reduzierung von Entnahmemengen bietet hier eine Möglichkeit zum Grundwassermanagement in der Zukunft. Die hohe Bedeutung der Grundwasserneubildung zeigen auch Woldeamlak et al. (2007) in Ihren Untersuchungen. Für das Einzugsgebiet der Grote/Nete (Belgien) wurden in zwei Steady-state-Szenarien klimabedingte Einflüsse auf das Grundwassersystem in den Jahren 2050 und 2100 simuliert. Hierbei stand eine Untersuchung des Einflusses verschiedener Parameter auf die Grundwasserneubildung für trockene und feuchte Klimaszenarien im Fokus. Es zeigte sich, dass die Grundwasserneubildung vor allem durch den Oberflächenabfluss und die Landnutzung und der hiermit einhergehenden Evapotranspiration beeinflusst wird.

Den Einfluss von Oberflächengewässern auf das Grundwasserregime wurde von Goderniaux et al. (2009) in einem gekoppelten Oberflächen- und Grundwassermodell untersucht. Auch wenn die Geologie anders ausgebildet ist, wird auch in dieser Untersuchung im belgisch-luxemburgischen Grenzgebiet von einer abnehmenden Grundwasserneubildung ausgegangen. Wie für die Fallstudie Bremerhaven ergeben sich für das Jahr 2040 zunächst geringe Auswirkungen, die sich in den Simulationsjahren 2070 und 2100 jedoch durch Rückgänge der Grundwasseroberfläche um bis zu $8 \mathrm{~m}$ zeigen. Goderniaux et al. (2009) weisen dabei insbesondere auf den Nutzen entsprechender Regionalmo- 
delle zum Grundwasserkörpermanagement über die Steuerung der Oberflächengewässer hin. Im Bereich des Untersuchungsgebietes Bremerhaven bietet insbesondere der Apeler See eine solche Möglichkeit des Grundwassermanagements. Der Wasserstand des Sees wird über ein Schöpfwerk gesteuert. Diese Entnahme im Abstrom des durch die Absenkung stark betroffenen Naturschutzgebietes wurde in den aktuellen Modellrechnungen noch konstant gehalten. Anpassungen an veränderte Klimabedingungen sind hier sicherlich sinnvoll und Diskussionspunkt in der Entwicklung einer Klimaanpassungsstrategie für die Stadt Bremerhaven. Dass regionale Strömungsmodelle für das Management von Grundwasserkörpern, insbesondere auch im Hinblick auf die Wasserrahmenrichlinie (Europäische Union 2000), einen bedeutenden Beitrag leisten können, wird auch von Højberg et al. (2007) unterstrichen. Als weitere Fortschreibung des Modells bietet sich eine Untersuchung der Auswirkung von prognostizierten Veränderungen in der Niederschlagsverteilung im Jahresverlauf an. Zusätzlich zu diesen intensiveren zeitlichen Untersuchungen sind auch für das Modell Bremerhaven Untersuchungen zu alternativen Klimaszenarien denkbar. In Verbindung mit einem Monitoring können die Modelle in der Folge kontinuierlich fortentwickelt und mit gemessenen Niederschlags- bzw. Neubildungsdaten abgeglichen werden.

Open Access. Dieser Artikel wird unter der Creative Commons Namensnennung 4.0 International Lizenz (http://creativecommons.org/ licenses/by/4.0/deed.de) veröffentlicht, welche die Nutzung, Vervielfältigung, Bearbeitung, Verbreitung und Wiedergabe in jeglichem Medium und Format erlaubt, sofern Sie den/die ursprünglichen Autor(en) und die Quelle ordnungsgemäß nennen, einen Link zur Creative Commons Lizenz beifügen und angeben, ob Änderungen vorgenommen wurden.

\section{Literatur}

Ad-hoc-AG Hydrogeologie: Regionale Hydrogeologie von Deutschland: die Grundwasserleitung: Verbreitung, Gesteine, Lagerungsverhältnisse, Schutz und Bedeutung. Geol. Jahrb. 163, 452 (2016)

Anderson, M.P., Woessner, W.: Applied groundwater modeling. Simulation of flow and advective transport. Academic Press, San Diego Calif (1992)

Barlow, P.M., Reichard, E.G.: Saltwater intrusion in coastal regions of North America. Hydrogeol. J. 18, 247-260 (2010)

Chang, S.W., Clement, T.P., Simpson, M.J., Lee, K.K.: Does sea-level rise have an impact on saltwater intrusion? Adv. Water Resour. 34, 1283-1291 (2011)

Dörhöfer, G., Hannappel, S., Voigt, H.-J.: Die Hydrogeologische Übersichtskarte von Deutschland (HÜK 200) (2001)

Elbracht, J., Meyer, R., Reutter, E.: Hydrogeologische Räume und Teilräume in Niedersachsen. GeoBerichte 3, 118 (2016)

Entenmann, W.: Hydrogeologische Untersuchungsmethoden von Altlasten. Springer, Berlin, Heidelberg, New York (1998)

Europäische Union: Richtlinie 2000/60/EG des europäischen Parlaments und des Rates vom 23. Oktober 2000 zur Schaffung eines Ordnungsrahmens für Maßnahmen der Gemeinschaft im Bereich der Wasserpolitik. Amtsblatt der Europäischen Gemeinschaften 327. (2000)
De Filippis, G., Borsi, I., Foglia, L., Cannata, M., Mansilla, V.V., Vasquez-Sune, E., Ghetta, M., Rossetto, R.: Software tools for sustainable water resources management: the GIS-integrated FREEWAT platform. Rend. Online Soc. Geol. Ital. 42, 59-61 (2017)

Fuchs, S.: Deterministische kf-Wert-Schätzung nach petrographischer Bohrgutansprache. Grundwasser 15(3), 177-189 (2010)

Goderniaux, P., Brouyere, S., Fowler, H.J., Blenkinsop, S., Therrien, R., Orban, P., Dassargues, A.: Large scale surface-subsurface hydrological model to assess climate change impacts on groundwater reserves. J. Hydrol. (Amst) 373, 122-138 (2009)

Hanson, R.T., Boyce, S.E., Schmid, W., Hughes, J.D., Mehl, S.M., Leake, S.A., Maddock III, T., Niswonger, R.G.: One-Water Hydrologic Flow Model (MODFLOW-OWHM). U.S. Geological Survey Techniques and Methods 6-A51. (2014)

Harbaugh, A.W.: A computer program for calculating subregional water budgets using results from the U.S. Geological Survey modular three-dimensional ground-water flow model. U.S. Geological Survey Open-File Report 90-392. (1990)

Harbaugh, A.W.: MODFLOW-2005, the U.S. geological survey modular ground-water model the ground-water flow process. U.S. Geological survey techniques and methods 6-A16. (2005)

Herrmann, F., Chen, S., Kunkel, R., Wendland, F.: Quantifizierung und Bewertung des innerjährlichen Abflussgeschehens und der Auswirkungen von Klimaänderungen auf den Wasserhaushalt in Niedersachsen und Bremen. Endbericht. Forschungszentrum Jülich, Jülich (2013)

Hoffmann, B., Meckelburg, M., Meinken, M.: Folgen einer Klimaänderung für den Grund- und Bodenwasserhaushalt der Unterwesermarsch. In: Schuchardt, B., Schirmer, M. (Hrsg.) Klimawandel und Küste: die Zukunft der Unterweserregion, S. 103-137. Springer, Berlin, Heidelberg (2006)

Højberg, A.L., Refsgaard, J.C., van Geer, F., Jørgensen, L.F., Zsuffa, I.: Use of models to support the monitoring requirements in the water framework directive. Water Resour. Manag. 21, 1649-1672 (2007)

IPCC: Climate Change: The physical science basis. Contribution of working group I to the fifth assessment report of the intergovernmental panel on climate change. In: Stocker, T.F. (Hrsg.) Fifth assessment report of the intergovernmental, Bd. 2013, Cambridge University Press, Cambridge (2013)

Jensen, H., Fritz, J., Bickelmann, H., Grützmann, J., Kantor, W., Kockel, F., Meißner, D., Reichling, J., Windelberg, S.: Grundwasserund Geotechnische Planungskarte Bremerhaven Geologischer Dienst für Bremen im Auftrag des Umweltschutzamtes der Seestadt Bremerhaven (2003)

Konikow, L.F., Kendy, E.: Groundwater depletion: a global problem. Hydrogeol. J. 13, 317-320 (2005)

Langevin, C.D., Thorne Jr., D.T., Dausman, A.M., Sukop, M.C., Weixing, G.: SEAWAT version 4: a computer program for simulation of multi-species solute and heat transport. U.S. Geological Survey Techniques and Methods Book 6. (2007). Chapter A22

Langguth, H.R., Voigt, R.: Hydrogeologische Methoden. Springer, Berlin, Heidelberg (2004)

Mallet, J. L.: gOcad: A computer-Aided Design Program for Geological Applications. In: Turner, K. (ed.), Three-Dimensional Modelling with Geoscientific Information Systems. Kluwer Academic Publishers Dordrecht Holland Nato ASI Series C132-141. (1992)

Panteleit, B., Jensen, S., Seiter, K., Budde, H., McDiarmid, J.: A regional geological and groundwater flow model of Bremen (Germany): an example management tool for resource administration. Z. Dtsch. Ges. Geowiss. 164, 569-580 (2013)

Poeter, E.P., Hill, M.C., Banta, E.R., Mehl, S., Christensen, S.: UCODE_2005 and six other computer codes for universal sensitivity analysis, calibration, and uncertainty evaluation. U.S. Geological Survey Techniques and Methods 6-A11. (2005)

Pollock, D.W.: User's guide for MODPATH/MODPATH-PLOT, version 3: a particle tracking post-processing package for MOD- 
FLOW, the US: geological survey finite-difference ground-water flow model. US Department of Interior, Washington D.C. (1994)

Post, V., Kooi, H., Simmons, C.: Using hydraulc head measurements in variable-density ground water flow analyses. Groundwater $\mathbf{4 5}$, 664-671 (2007)

QGIS: QGIS geographic information System. Open source geospatial Foundation project (2015)

Richey, A.S., Thomas, B.F., Lo, M.H., Reager, J.T., Famiglietti, J.S., Voss, K., Swenson, S., Rodell, M.: Quantifying renewable groundwater stress with GRACE. Water Resour. Res. 51, 5217-5238 (2015a)

Richey, A.S., Thomas, B.F., Lo, M.H., Famiglietti, J.S., Swenson, S., Rodell, M.: Uncertainty in global groundwater storage estimates in a Total Groundwater Stress framework. Water Resour. Res. 51, 5198-5216 (2015b)

Schirmer, M.: Das Klimaszenario der Fallstudie „Klimaänderung und Unterweserregion“ (KLIMU). In: Schuchardt, B., Schirmer, M. (Hrsg.) Klimawandel und Küste: die Zukunft der Unterweserregion, S. 49-56. Springer, Berlin, Heidelberg (2006)
Stocker, T.F., Qin, D., Plattner, G.-K., Tignor, M., Allen, S.K., Boschung, J., Nauels, A., Xia, Y., Bex, B., Midgley, B.: IPCC: The physical science basis. Contribution of working group I to the fifth assessment report of the intergovernmental panel on climate change. Cambridge University Press, Cambridge (2013)

Woldeamlak, S.T., Batelaan, O., de Smedt, F.: Effects of climate change on the groundwater system in the Grote-Nete catchment, Belgium. Hydrogeol. J. 15, 891-901 (2007)

Graf Yang, J., Graf, T., Ptak, T.: Impact of climate change on freshwater resources in a heterogeneous coastal aquifer of Bremerhaven, Germany: A three-dimensional modeling study. J. Contam. Hydrol. 177, 107-121 (2015)

Zheng, C., Wang, P.P.: MT3DMS, A modular three-dimensional multi-species transport model for simulation of advection, dispersion and chemical reactions of contaminants in groundwater systems; documentation and user's guide. U.S. Army Engineer Research and Development Center Contract Report SERDP-99-1. U.S. Army Engineer Research and Development Center, Vicksburg (1999) 